\title{
Interest Rate Risk Management and Dynamic Portfolio Selections
}

\author{
Hang Sun ${ }^{1}$, Wan-gui Sun ${ }^{2}$ \\ ${ }^{1}$ School of Finance \& Taxation, Southwest University of Finance and Economics, Chengdu, China \\ ${ }^{2}$ School of Economics \& Management, Northwest University, Xi' an, China \\ E-mail: sunhang728@163.com,wgsun@126.com \\ Received May 9, 2011; revised July 2, 2011; accepted July 13, 2011
}

\begin{abstract}
The dynamic portfolio selections in the sense of Markowitz's mean-variance are addressed in an incomplete market and the effect of interest rate risk on them is discussed. According to Markowitz's measure risk approach, the interest rate risk is divided into the controllable risk and the uncontrollable risk. The former can be hedged, but the latter cannot. The zero-coupon bond is an efficient tool to avoid the interest rate risk. The optimal payoff resulting from self-financed strategies and the mean-variance efficient frontier are expressed explicitly. The results show that the optimal payoff and the efficient frontier are not affected by the controllable risk of interest rate, but by the uncontrollable risk. The efficient frontier is a part of a hyperbola if there exists the uncontrollable risk. The expected optimal payoff grows with the increase of risk; however, the margin expected optimal payoff lowers. The efficient frontier is a straight line if and only if there is no uncontrollable risk.
\end{abstract}

Keywords: Portfolio Selection, Efficient Frontier, Interest Rate Risk, Incomplete Market

\section{Introduction}

The portfolio problem is one of major issues in applied and theoretical finance. And the interest rate is an important factor in portfolio selections and a main reason why the global financial crisis took place in 2008. Markowitz [1] provides a powerful framework for assessing the riskreturn of assets and the benefit of diversification. Optimal multiperiod portfolios are characterized in Merton [2]. And then dynamic mean-variance portfolios are characterized in Richardson [3] for a fairly simple investment opportunity set, and in Bajeux-Besnainou and Portait [4] for more complex asset price dynamics. Taking advantage of the general stochastic linear-quadratic theory, Zhou and Li [5] and Lim and Zhou [6] gave an analytical optimal portfolio strategy and an explicit expression of the efficient frontier in the case of a continuous-time mean-variance portfolio selection problem in a complete market. The typical examples of the incomplete market were studied on claim hedging in Karatzas [7] and $\mathrm{He}$ and Pearson [8], where the number of stocks is not greater than the number of Brownian motions in the price processes of stocks. The purpose of this paper is to address the dynamic mean-variance portfolio selections in the incomplete market with interest rate risk.

The optimal payoff and the mean-variance efficient frontier are expressed in a financial background with respect to the variance-optimal martingale measures introduced in Schweizer [9] or [10]. In general, the efficient frontier is a part of a hyperbola. With the increase of investors' risk, their expected return grows while the margin expected return lowers.

The reasons for the non-straight-line efficient frontier are both the interest rate risk and market incompleteness. It is proved that the efficient frontier is a straight line under the assumption that either the interest rates are determined or the market is complete.

The effect of the interest rate risk on portfolio selection is analyzed. The interest rate risk is divided into the controllable risk and the uncontrollable risk. The controllable risk, related to capital market, can be hedged. The expected return produced by the controllable risk may be positive, zero or negative. On the contrary, the uncontrollable risk, having no relevance to capital market, cannot be hedged. The latter affects investors' expected return, but the former does not. The resolution of the interest rate risk is different from that by Markowitz. A reasonable interpretation for the optimal payoff and the 
efficient frontier are given with the resolution. And both of them are not affected by the controllable risk, but by the uncontrollable risk. The efficient frontier is a line if and only if the uncontrollable risk does not exist

The paper is structured as follows. The next section presents the model we used and the financial background. In section 3, we introduce several lemmas and the relations between the variance-optimal martingale measures and the orthogonal projections. Section 4 presents the solution to our problem and analyzes the meanings of its solution. We determine the mean-variance efficient frontier and conclude our paper in the last section.

\section{Model and Financial Background}

Let $(\Omega, F, P)$ be a complete probability space and $L^{2}(\Omega, F, P)$ be the space of all square-integrable real random variables with scalar product $\langle X, Y\rangle=E[X Y]$ and norm $\|X\|=\sqrt{E\left[X^{2}\right]}$. Let $M$ be a linear subspace of $L^{2}(\Omega, F, P)$. A given element $B \in L^{2}(\Omega, F, P)$ satisfies $B>0 P-$ a.s. and $B \notin M$.

The pair $(M, B)$ represents the financial environment in which the subsequent considerations take place. $M$ corresponds to a financial market without frictions, such as transaction costs, constraints or other restrictions on strategies. An element $m$ of $M$ models the total gains from trade resulting from a self-financing trading strategy with initial capital $0 . B$ is interpreted as the final value of some bond with initial value 1 . In particular, the case $B=1$ corresponds to interest rate 0 .

An example: Considering a finite time span $[0, T]$, Let $(\Omega, F, P)$ be a probability space with a filtration $\left(F_{t}\right)(t \in[0, T])$ satisfying the usual conditions of rightcontinuity and completeness. We also assume that $F_{0}$ is trivial and $F_{T}=F$. There exist $N+1$ primitive assets comprising the $R^{N+1}$ - valued price process $\left(S^{0}, S\right)$ : one bond whose price process is given by $S_{t}^{0}=\exp \left\{\int_{0}^{t} r(s) \mathrm{d} s\right\}$, where $r$ is a progressively measurable process with respect to $\left(F_{t}\right)$, interpreted as the instantaneous interest rate, and $N$ risky assets, whose $R^{N}-$ valued price process $S_{t} \equiv\left(S_{1}(t), \cdots, S_{N}(t)\right)$ is a continuous semimartingale. As in [11] and [12], we introduce two sets

$$
\begin{aligned}
& \mathrm{M}_{2}= \\
& \left\{Q<<: \frac{1}{S_{T}^{0}} \frac{\mathrm{d} Q}{\mathrm{~d} P} \in L^{2}(P), S / S^{0} \text { is a } Q \text { - local martingale }\right\}, \\
& \mathrm{M}_{2}^{e}= \\
& \left\{Q \sim P: \frac{1}{S_{T}^{0}} \frac{\mathrm{d} Q}{\mathrm{~d} P} \in L^{2}(P), S / S^{0} \text { is a } Q \text { - local martingale }\right\},
\end{aligned}
$$

and two standing assumptions: Assumption 1. $\left|\int_{0}^{T} r(t) \mathrm{d} t\right| \leq L, P$ a.s. for some positive constant $L$;

Assumption 2. $\mathrm{M}_{2}^{e} \neq \phi$. Then we define the space of trading strategies $\theta$. Given a real-valued adapted process $V$ and $R^{N}$-valued predictable process, we can define a portfolio of market value $V$ with quantities $\theta$, $\theta^{0}$ in the primitive assets $S, S^{0}$, satisfying $V=\theta^{0} S^{0}+\theta^{\prime} S$ and $\theta_{t}^{o}=\left(V_{t}-\vartheta_{t}^{\prime} S_{t}\right) / S_{t}^{0}$. Portfolio $(V, \theta)$ is called self-financed with respect to the primitive assets $\left(S^{0}, S\right)$ if $\theta \in \Theta$ and

$$
\mathrm{d} V_{t}=\theta_{t}^{o} \mathrm{~d} S_{t}^{0}+\theta_{t}^{\prime} \mathrm{d} S_{t}
$$

where $\Theta$ is the set of $R^{n}$-valued $S / S^{0}$ - integrable predictable processes $\theta$ such that

$S_{T}^{0} \int_{0}^{T} \theta_{t}^{\prime} \mathrm{d} \frac{S_{t}}{S_{t}^{0}} \in L^{2}(P)$, and for each $Q \in \mathrm{M}_{2}$, the process $\int_{0}^{T} \theta_{t}^{\prime} \mathrm{d} \frac{S_{t}}{S_{t}^{0}}$ is a Q-martingale. From relation (1), $V_{T}=S_{T}^{0}\left(V_{0}+\int_{0}^{T} \theta_{t}^{\prime} \mathrm{d} \frac{S_{t}}{S_{t}^{0}}\right)$. Let $B \equiv S_{T}^{0}$ and $M \equiv\left\{m=S_{T}^{0} \int_{0}^{T} \theta_{t}^{\prime} \mathrm{d} \frac{S_{t}}{S_{t}^{0}} ; \theta \in \Theta\right\}$.

$(M, B)$ satisfies our assumptions, i.e., $M$ is a linear subspace of $L^{2}(\Omega, F, P)$ and $B \notin M$.

In view of Markowitz's mean-variance point [1], given a constant expected return, select optimal portfolio such that the risk of the portfolio is minimal. Thus consider the following problem:

$$
\text { (P) }\left\{\begin{array}{l}
\min _{m \in M} \operatorname{Var}\left[V_{T}\right] \\
\text { s.t. } E\left[V_{T}\right]=\bar{V} .
\end{array}\right.
$$

where $V_{T}=V_{0} B+m$ and $\bar{V}$ is a real constant. The solution of problem $(\mathrm{P})$ is expressed by $V_{T}^{*}$. Point $\left(\sqrt{\operatorname{Var}\left[V_{T}^{*}\right]}, \bar{V}\right) \in R^{2}$ is call mean-variance efficient. The mean-variance efficient frontier is the set of all meanvariance efficient points.

In the preceding example, problem $(\mathrm{P})$ is equivalent to the following problem:

$$
\text { (P') }\left\{\begin{array}{l}
\min _{\theta \in \Theta} \operatorname{Var}\left[V_{T}\right] \\
\text { s.t. } E\left[V_{T}\right]=\bar{V} .
\end{array}\right.
$$

where $V_{T}=S_{T}^{0} V_{0}+S_{T}^{0} \int_{0}^{T} \theta_{t}^{\prime} \mathrm{d} \frac{S_{t}}{S_{t}^{0}}$ and $\bar{V}$ is a real constant.

In this paper, we address problem $(\mathrm{P})$ under general assumptions (such as incomplete markets and stochastic interest rates) and our results apply to portfolios of 
claims in a single period, multiperiod and continuous time. As mentioned in the introduction, Richardson [3] and Bajeux-Besnainou and Portait [4] solve the problem (P') in the complete market by applying the method of dynamic programming. Instead of the dynamic stochastic control theory, Zhou and Li [5] and Lim and Zhou [6] discussed the same problem under market completeness. Although Sun and Wang [13] analyzed a similar problem, the original probability is replaced by an equivalent one, so called $P^{B}$, and the interest rate risk is omitted.

\section{Methodology}

In this section an auxiliary problem is introduced and the relation between the auxiliary problem and problem (P) is discussed. And some lemmas and notions are presented. It should be mentioned that the orthogonal projection is an important tool to solve our problems.

Problem (P) can be solved via the following utility optimization problem:

$$
\text { (P*) } \sup _{m \in M} u\left(V_{T}\right)
$$

with the quasi-quadratic utility function $u(Y)$ defined by $u(Y)=E[Y]-A \operatorname{Var}[Y], A>0$ is a risk aversion parameter.

The relation between problems $(\mathrm{P})$ and $\left(\mathrm{P}^{*}\right)$ is described as the following.

Lemma 1: If $V_{T}^{*}$ is a solution to problem $\left(\mathrm{P}^{*}\right)$ then point $\left(\sqrt{\operatorname{Var}\left[V_{T}^{*}\right]}, E\left[V_{T}^{*}\right]\right)$ is mean-variance efficient.

Proof: Let $V_{T}^{*}$ be a solution to problem $\left(\mathrm{P}^{*}\right)$ and select parameter $A$ such that $\bar{V}=E\left[V_{T}^{*}\right]$. For each $m \in M$ satisfying equation $E\left[V_{T}\right]=\bar{V}$, we follow that $u\left(V_{T}\right) \leq u\left(V_{T}^{*}\right)$. Thus $\operatorname{Var}\left[V_{T}^{*}\right] \leq \operatorname{Var}\left[V_{T}\right]$, which means the lemma holds true. Q.E.D.

Lemma 1 shows that the quasi-quadratic utility exhibits the mean-variance efficiency. Next we present some other lemmas for the solutions to problems $(\mathrm{P})$ and $\left(\mathrm{P}^{*}\right)$.

Lemma 2: If $Y \in L^{2}(\Omega, F, P)$ then

$$
\operatorname{Var}[Y]=E\left[(Y-E[Y])^{2}\right]=\inf _{a \in R} E\left[(Y-a)^{2}\right] \text {. }
$$

Lemma 3: $\inf _{m \in M} \operatorname{Var}[B-m]=\inf _{c \in R} \inf _{m \in M} E\left[(B-m-c)^{2}\right]$. And if $m^{*}(c)$ and $c^{*}$ are the solutions to, respectively, $\inf _{m \in M} E\left[(B-m-c)^{2}\right]$ and $\inf _{c \in R} E\left[\left(B-m^{*}(c)-c\right)^{2}\right]$, then $m^{*}\left(c^{*}\right)$ is the solution to $\inf _{m \in M} \operatorname{Var}[B-m]$.

Proof: From Lemma $2 \operatorname{Var}[B-m] \leq E\left[(B-m-c)^{2}\right]$ for each $c \in R$. Thus

$$
\inf _{m \in M} \operatorname{Var}[B-m] \leq \inf _{c \in R} \inf _{m \in M} E\left[(B-m-c)^{2}\right] .
$$

On the other hand, let $m^{*}$ be the solution to $\inf _{m \in M} \operatorname{Var}[B-m]$. Then

$$
\begin{aligned}
& \inf _{m \in M} \operatorname{Var}[B-m]=\operatorname{Var}\left[B-m^{*}\right] \\
& =E\left[\left(B-m^{*}-c^{*}\right)^{2}\right] \geq \inf _{c \in R} \inf _{m \in M} E\left[(B-m-c)^{2}\right]
\end{aligned}
$$

The first part of the lemma holds. And the second can be seen from the proof above. Q.E.D.

Lemma 4: If the solution to $\sup _{a \in R} \sup _{m \in M}\left\{E\left[V_{0} B+m\right]-A E\left[V_{0} B+m-a\right]^{2}\right\}$ exists (such as $\left.a^{*} \in R, m^{*}(a) \in M\right)$, then the follow relation holds:

$$
\begin{aligned}
& \sup _{m \in M}\left\{E\left[V_{0} B+m\right]-A \operatorname{Var}\left[V_{0} B+m\right]\right\} \\
& =\sup _{a \in R} \sup _{m \in M}\left\{E\left[V_{0} B+m\right]-A E\left[V_{0} B+m-a\right]^{2}\right\}
\end{aligned}
$$

And $m^{*}\left(a^{*}\right) \in M$ is the solution to problem $\sup _{m \in M}\left\{E\left[V_{0} B+m\right]-A \operatorname{Var}\left[V_{0} B+m\right]\right\}$ with $a^{*}=E\left[m^{*}\left(a^{*}\right)\right]$

The proof of lemma 4 is similar to that of lemma 3 .

The orthogonal projections $\pi(B)$ and $\pi(1)$ are closely related to the problems

$$
\text { (W) } \min _{m \in M} E\left[(B-m)^{2}\right]
$$

and

$$
\text { (W') } \min _{m \in M} E\left[(1-m)^{2}\right] \text {. }
$$

The two problems have solutions $B-\pi(B)$ and $1-\pi(1)$, respectively, and valves $E\left[(\pi(B))^{2}\right.$ and $E[\pi(1)]$. Note that $1 \notin M$ by no-arbitrage condition. It implies $\pi(1) \neq 0$ and $E[\pi(1)]>0$ (as shown in [10]).

The orthogonal projections are also related to variance-optimal martingale measures closely. The two variance-optimal martingale measures $\tilde{P}_{\mathrm{B}}$ and $\tilde{P}_{1}$, respectively, can be defined in the example above as the solutions to the problems

$$
\text { (D) } \min _{Q \in \mathrm{M}_{2}} E\left[\left(\frac{1}{B} \frac{\mathrm{d} Q}{\mathrm{~d} P}\right)^{2}\right]
$$

and

$$
\text { (D') } \min _{Q \in \mathrm{M}_{2}} E\left[\left(\frac{\mathrm{d} Q}{\mathrm{~d} P}\right)^{2}\right] \text {. }
$$

Problems (D) and (D') refer Schweizer [9] and [10], Delbaen and Schachermayer [11], Gourieroux et al. [12] and Laurent and Pham [14]. We can express projections $\pi(B)$ and $\pi(1)$ with the measures $\tilde{P}_{\mathrm{B}}$ and $\tilde{P}_{1}$. Under the assumptions 1 and 2 in the example, we have

$$
\pi(B)=\frac{1}{B} \frac{\mathrm{d} \tilde{P}_{B}}{\mathrm{~d} P} / E\left(\frac{1}{B} \frac{\mathrm{d} \tilde{P}_{B}}{\mathrm{~d} P}\right)^{2}
$$


and

$$
\pi(1)=\frac{\mathrm{d} \tilde{P}_{1}}{\mathrm{~d} P} / E\left(\frac{\mathrm{d} \tilde{P}_{1}}{\mathrm{~d} P}\right)^{2}
$$

And the measures $\tilde{P}_{\mathrm{B}}$ and $\tilde{P}_{1}$ are equivalent to measure $P$.

\section{Solutions and Analysis}

The solutions to problem $\left(\mathrm{P}^{*}\right)$ and then problem $(\mathrm{P})$ are given and the meanings of their solutions are analyzed in this section.

Proposition 1: The solution to problem $\left(\mathrm{P}^{*}\right)$ is given below

$$
\begin{aligned}
& m^{*}=V_{0}\left[-(B-\pi(B))+\frac{E[\pi(B)]}{E[\pi(1)]}(1-\pi(1))\right] \\
& +\frac{1}{2 A} \frac{(1-\pi(1))}{E[\pi(1)]}
\end{aligned}
$$

Proof: From lemma 4, we have

$$
\sup _{m \in M} u\left(V_{T}\right)=\sup _{a \in R} \sup _{m \in M}-A E\left[\left(V_{0}(B-\pi(B))+m-\left(a+\frac{1}{2 A}\right)(1-\pi(1))+V_{0} \pi(B)-\left(a+\frac{1}{2 A}\right) \pi(1)\right)^{2}\right]+\frac{1}{4 A}+a
$$

since $V_{0}(B-\pi(B))+m-\left(a+\frac{1}{2 A}\right)(1-\pi(1)) \in M \quad$ and $V_{0} \pi(B)-\left(a+\frac{1}{2 A}\right) \pi(1)$ are orthogonal to $M$, we follow

$$
\begin{aligned}
& \sup _{m \in M} u\left(V_{0} B+m\right) \\
& =\sup _{a \in R}-A E\left[\left(V_{0} \pi(B)-\left(a+\frac{1}{2 A}\right) \pi(1)\right)^{2}\right]+\frac{1}{4 A}+a
\end{aligned}
$$

as taking $m^{*}=-V_{0}(B-\pi(B))+(a+1 / 2 A)(1-\pi(1))$. Thus the original problem is transformed to an optimization problem with a quadratic function. It has solution

$$
a^{*}=\left(V_{0} E[\pi(B)]+\frac{1}{2 A} E[1-\pi(1)]\right) / E[\pi(1)] .
$$

Substituting $a$ for $a^{*}$ in $m^{*}$, we know the proposition true from lemma 4. The proof is complete. Q.E.D.

Proposition 2: The solution to problem $(\mathrm{P})$ is given as follow:

$$
\begin{aligned}
& V_{T}^{*}=V_{0} B+V_{0}\left[-(B-\pi(B))+\frac{E[\pi(B)]}{E[\pi(1)]}(1-\pi(1))\right] \\
& +\left[\bar{V}-\frac{V_{0} E[\pi(B)]}{E[\pi(1)]}\right] \frac{(1-\pi(1))}{1-E[\pi(1)]}
\end{aligned}
$$

for $E[\pi(1)] \neq 1$ and $\bar{V}>\frac{V_{0} E[\pi(B)]}{E[\pi(1)]} ; V_{T}^{*}=V_{0} B$ for $E[\pi(1)]=1$ and $\bar{V}=V_{0} E[\pi(B)]$.

Proof: From proposition 1 and lemma 1, the optimal payoff is

$$
\begin{aligned}
m^{*}= & V_{0}\left[-(B-\pi(B))+\frac{E[\pi(B)]}{E[\pi(1)]}(1-\pi(1))\right] \\
& +\frac{1}{2 A} \frac{(1-\pi(1))}{E[\pi(1)]}
\end{aligned}
$$

if $E[\pi(1)] \neq 1$ and $\bar{V}>\frac{V_{0} E[\pi(B)]}{E[\pi(1)]}$. Adding $V_{0} B$ in both sides and taking expectation, we know

$$
E\left[V_{0} B+m^{*}\right]=\frac{V_{0} E[\pi(B)]}{E[\pi(1)]}+\frac{1}{2 A} \frac{1-E[\pi(1)]}{E[\pi(1)]}=\bar{V} .
$$

Thus

$$
\frac{1}{2 A}=\left[\bar{V}-\frac{V_{0} E[\pi(B)]}{E[\pi(1)]}\right] \frac{E[\pi(1)]}{1-E[\pi(1)]}
$$

Substituting in relation (4), the first part of the proposition is true.

Now we turn to the second one. The solution to problem $(\mathrm{P})$ is

$V_{T}^{*}=V_{0} B-V_{0}(B-\pi(B))+\left(V_{0} E[\pi(B)]+\frac{1}{2 A}\right)(1-\pi(1))$

if $E[\pi(1)]=1$ and $\bar{V}=V_{0} E[\pi(B)]$. Relation

$E[\pi(1)]=1$ implies $\pi(1)=1$. Thus $V_{T}^{*}=V_{0} B$ if

$E[\pi(1)]=1$ and $\bar{V}=V_{0} E[\pi(B)]$. We complete the proof. Q.E.D.

Next, consider problem

$$
\inf _{m \in M} \operatorname{Var}[B-m],
$$

that is, seeking payoff $m$ by a self-financed strategy such that a bond holder has minimal risk (by short-selling stocks with payoff $\mathrm{m}$ ).

Proposition 3: The solution to problem $\inf _{m \in M} \operatorname{Var}[B-m]$ is given by

$$
m^{*}=(B-\pi(B))-\frac{E[\pi(B)]}{E[\pi(1)]}(1-\pi(1)) .
$$

Proof: From lemma 3, one has 


$$
\begin{aligned}
& \inf _{m \in M} \operatorname{Var}[B-m]= \\
& \inf _{c \in R} \inf _{m \in M} E\left[(\pi(B)+(B-\pi(B))-m-c(1-\pi(1))-c \pi(1))^{2}\right]
\end{aligned}
$$

Because $\pi(B)$ and $\pi(1)$ are orthogonal to $M$, we have

$$
\begin{aligned}
& \inf _{m \in M} \operatorname{Var}[B-m]=\inf _{c \in R} E\left[(\pi(B)-c \pi(1))^{2}\right] \\
& =\inf _{c \in R} E\left[(\pi(B))^{2}\right]-2 c E[\pi(B)]+c^{2} E[\pi(1)]
\end{aligned}
$$

by taking $m^{*}(c)=(B-\pi(B))-c(1-\pi(1))$. The last optimization problem has solution $c^{*}=\frac{E[\pi(B)]}{E[\pi(1)]}$.

Again from lemma 3, $m^{*}\left(c^{*}\right)$ is a solution to problem $\inf _{m \in M} \operatorname{Var}[B-m]$. Q.E.D.

Bond payoff $B$ can be rewritten as $B=\bar{B}+(B-\bar{B})$, where

$$
\bar{B}=\pi(B)+\frac{E[\pi(B)]}{E[\pi(1)]}(1-\pi(1)) .
$$

From proposition $3, B-\bar{B}$ is the optimal replicable part of $B$ in the sense of minimal risk, and $\bar{B}$ is the leftover part. $\bar{B}$ and $B-\bar{B}$ is called, respectively, as the uncontrollable payoff and the controllable one of $B$. Their expectations are referred as the expected uncontrollable payoff and the expected controllable payoff, and their variances as the uncontrollable risk and the controllable risk of $B$.

The resolution of interest rate risk gives a reasonable interpretation of the solutions to problems $\left(\mathrm{P}^{*}\right)$ and $(\mathrm{P})$. The solution to problem $\left(\mathrm{P}^{*}\right)$ has two terms: one payoff by a short-selling self-financed strategy to avoid the controllable risk of interest rates; and the other by a self-financed strategy dependent on investors' risk preference.

\section{Results and Conclusions}

At last, we determine the mean-variance efficient frontier, present its properties and conclude our paper.

Let $V_{T}^{*}=m^{*}+V_{0} B$. Form proposition 1, we get

$$
E\left[V_{T}^{*}\right]=V_{0} E[\bar{B}]+\frac{1}{2 A} \frac{E[1-\pi(1)]}{E[\pi(1)]}
$$

and

$$
\operatorname{Var}\left[V_{T}^{*}\right]=V_{0}^{2} \operatorname{Var}[\bar{B}]+\frac{1}{4 A^{2}} \frac{E[1-\pi(1)]}{E[\pi(1)]}
$$

Thus we have

$$
\begin{aligned}
& E\left[V_{T}^{*}\right]=V_{0} E[\bar{B}] \\
& +\sqrt{\frac{E[1-\pi(1)]}{E[\pi(1)]}} \cdot \sqrt{\operatorname{Var}\left[V_{T}^{*}\right]-V_{0}^{2} \operatorname{Var}[\bar{B}]}
\end{aligned}
$$

Proposition 4: The mean-variance efficient frontier of problem (P) is given by relation (6).

Obviously, the expected optimal payoff is not affected by the controllable risk, but by the uncontrollable risk of interest rates. If one only takes the uncontrollable risk $\operatorname{Var}[\bar{B}]$, her or his expected return is $V_{0} E[\bar{B}]$, the expected uncontrollable return. Define the excess expected return as $\left(E\left[V_{T}^{*}\right]-V_{0} E[\bar{B}]\right)$, the difference between the expected optimal return and the expected uncontrollable return, the excess risk as

$\sqrt{\operatorname{Var}\left[V_{T}^{*}\right]-V_{0}^{2} \operatorname{Var}[\bar{B}]}$, the square root of the difference between the variance of the optimal payoff and the uncontrollable risk of interest rates $V_{0}^{2} \operatorname{Var}[\bar{B}]$, and the excess risk price as $\sqrt{E[1-\pi(1)] / E[\pi(1)]}$. The product of the excess risk price and the excess risk $\sqrt{E[1-\pi(1)] / E[\pi(1)]} \cdot \sqrt{\operatorname{Var}\left[V_{T}^{*}\right]-V_{0}^{2} \operatorname{Var}[\bar{B}]}$ can be seen as the excess premium. Thus the excess expected return is equal to the excess premium.

If the uncontrollable risk $\operatorname{Var}[\bar{B}]$ is zero, the excess risk, the excess risk price and the excess risk premium are the standard deviation of the optimal payoff, the risk price and the risk premium, respectively. In this case, the efficient frontier is a line

$$
E\left[V_{T}^{*}\right]=V_{0} E[\bar{B}]+\sqrt{\frac{E[1-\pi(1)]}{E[\pi(1)]}} \cdot \sqrt{\operatorname{Var}\left[V_{T}^{*}\right]},
$$

which is usually called as the capital market line.

The following proposition further discusses the efficient frontier.

Proposition 5: The following statements are equivalent:

1) The efficient frontier is a line;

2) $\operatorname{Var}[\bar{B}]=0$;

3) $\bar{B}$ is determined;

4) $\varepsilon=0$;

5) There exists a real number $\alpha$ such that $\pi(B)=\alpha \pi(1)$;

6) $1 \in R B+M$.

Proof: It is clear that the conditions a) and b) are equivalent and that condition b) is equivalent to c) and d). From relation (5) and Holder's inequity, condition b) is equivalent to e). At last, we prove b) and e) are equivalent. On one hand, condition e) implies f) since $1=\pi(1)+[1-\pi(1)], \quad[1-\pi(1)] \in M$ and $\alpha \neq 0$. On the other hand, if f) holds, then there exist a real number 
$\gamma$ and $m \in M$ such that $1=\gamma B+m$. Thus

$\pi(1)=\gamma \pi(B)+\pi(m)=\gamma \pi(B)$. Note that $\pi(1)$ and $\pi(B)$ are not zero. It follows that $\gamma \neq 0$, that is, e) holds. The proof completes. Q.E.D.

The relation $1 \in R B+M$ means that the zero-coupon bond is an asset (or derived asset) in the market. From proposition 5, the zero-coupon bond is a useful tool to avoid the interest rate risk, including not only the controllable risk but also the uncontrollable risk.

Comparatively, Bajeux-Besnainou and Portait [4] proved the mean-variance efficient frontier is a line (an explicit expression is given only in a complete market) in a market with a bond, a zero-coupon bond and some stocks, no matter whether it is complete or not.

In the two particular cases, determined interest rates or complete markets, the efficient frontier is a straight line. For constant $B, \bar{B}=B$ and then the efficient frontier can be simplified as

$$
E\left[V_{T}^{*}\right]=V_{0} B+\sqrt{\frac{E[1-\pi(1)]}{E[\pi(1)]}} \cdot \sqrt{\operatorname{Var}\left[V_{T}^{*}\right]} .
$$

And for a complete market, the dimension of $M^{\perp}$ is one. It follows there exists a constant $\gamma$ satisfying $\pi(B)=\gamma \pi(1)$, which implies a linear efficient frontier as shown by relation (7).

In short, the uncontrollable risk of interest rate affects both the optimal payoff and the efficient frontier in the mean-variance portfolio selections. And the risk can be avoided in the use of the zero-coupon bond.

\section{Acknowledgements}

We would like to thank an anonymous referee of this journal for comments and suggestions which we believe have led to a much better paper.

\section{References}

[1] H. Markowitz, "Portfolio Selection," Journal of Finance, Vol. 7, No. 1, 1952, pp. 77-91.
[2] R. Merton, “An Intertemporal Capital Asset Pricing Model", Econometrica, Vol. 41, No. 5, 1971, pp. 867-888.

[3] H. Richardson, "A Minimum Variance Result in Continuous Trading Portfolio Optimization," Management Science, Vol. 35, No. 3, 1989, pp. 1045-1055.

[4] I. Bajeux-Besnainou and R. Portait, "Dynamic Asset Allocation in a Mean-Variance Framework," Management Science, Vol. 44, No. 11, 1998, pp. 79-95.

[5] X. Y. Zhou and D. Li, "Continuous-Time Mean-Variance Portfolio Selection: a Stochastic LQ Framework," Applied Mathematics \& Optimization, Vol. 42, No. 1, 2000, pp. 19-33.

[6] A. E. B. Lim and X. Y. Zhou, "Mean-Variance Portfolio Selection with Random Parameters in a Complete Market," Applied Mathematics \& Optimization, Vol. 27, No. 1, 2002, pp. 101-120.

[7] I. Karatzas, J. Lehoczky, S. E. Shreve and G. Xu, "Martingale and Duality Methods for Utility Maximization in an Incomplete Market," SIAM Journal of Control and Optimization, Vol. 29, No. 1, 1991, pp. 702-730.

[8] H. He and N. D. Pearson, "Consumption and Portfolio Policies with Incomplete Markets and Short-Sale Constraints," Journal of Economic Theory, Vol. 54, No. 8, 1991, pp. 259-305.

[9] M. Schweizer, "Approximation Pricing and the Variance-Optimal Martingale Measure," Annals of Probability, Vol. 24, No. 1, 1996, pp. 206-236.

[10] M. Schweizer, "From Actuarial to Financial Valuation Principles," Insurance: Mathematics \& Economics, Vol. 28, No. 1, 2001, pp. 31-47.

[11] F. Delbaen and W. Schachermayer, "The Variance-OptiMal Martingale Measure for Continuous Processes," Bernoulli, Vol. 2, No. 1, 1996, pp. 81-105.

[12] C. Goureroux, J. P. Laurent and H. Pham, "Mean-Variance Hedging and Numeraire," Mathematical Finance, Vol. 8, No. 3, 1998, pp. 179-200.

[13] W. G. Sun and C. F. Wang, "The Mean-Variance InvestMent Problem in a Constrained Financial Market," Journal of Mathematical Economics, Vol. 42, No. 11, 2006, pp. 885-895.

[14] J. P. Laurent and H. Pham, "Dynamic Programming and Mean-Variance Hedging," Finance and Stochastics, Vol. 3, No. 1, 1999, pp. 83-110. 